\title{
AN EXPERIMENT ON INTERSTRAND COUPLING CURRENTS IN SOLDERED CABLES
}

L. J.M van de Kl undert, R. A. Hartmann, H.G. Knoopers, A. Nijhuis, A.A. Oldenburger

University of Twente

Department of Applied Physics

P. O. Box 217, 7500 AE Enschede, the Netherlands.

Abstract: The interstrand coupling currents may in many cases produce the major part of the loss in cables subject to AC fields or carrying an AC transport current. In this paper the effective frequency dependent transverse resistivity experienced by the coupling currents is studied in an experimental way. The results are compared with simple mathematical considerations. An accompanying effect is a transverse magnetization of the cable strands due to the transverse current. This magnetization produces in the time dependent case eddy currents along the strands which enhances the effective AC transverse resistivity. The mathematical considerations include the dependency of the total loss as a function of frequency and the twist length of the wires.

\section{Introduction}

Calculation of the interstrand coupling loss in superconducting cables requires the knowledge of the constitutive equations concerning current and electric field in the direction parallel to the strand and transverse to it. The latter, transverse resistivity has already been subject to prior studies $[1,2,3]$ in the $\mathrm{DC}$ case. It was shown there that due to the filament twisting a reduction of the transverse resistivity occurs. The loss calculations, mentioned above, play an important role in cable design and in the interpretation of experimental results.

The twisting of the filaments of the strands results also in a magnetic effect: when a transport current flows in a twisted wire a B-component parallel to the wire axis occurs. On the other hand, if a current transverse to the wire is applied, the current in the filament region shall flow in the filament direction and consequently will have components in the direction of the wire axis with opposite directions in the upper and lower half of the wire, see fig. 1. From these currents a magnetic field in the direction perpendicular to the wire is generated, which is parallel to the current in case of counterclockwise

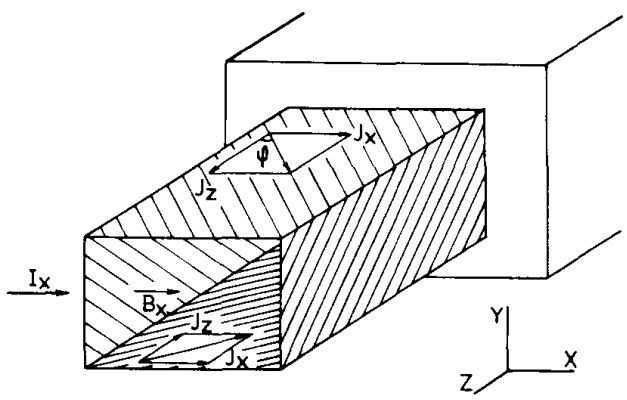

Fig. 1 Schematic view of the rectangular wire with the filament layer.

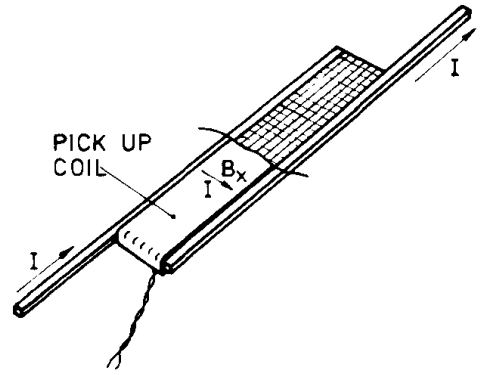

Fig. 2 Test arrangement with 6 parallel wires divided in 32 parts. Also the pick up coil for $\widetilde{B}_{x}$ is shown in part.

twisting and antiparallel in case of clockwise twisting. The field strength of this magnetic field depends on the twist length and is zero if no twist is present. It should be emphasized that small transverse currents depending on the twistlength (or twist angle $\varphi$ at the edge of filament region) can produce large currents parallel to the wire axis so saturating the filaments in case of a transport current is also present. In this paper we will assume that no saturation occurs and omit all non-linear effects associated with it.

For our experiments we choose a wire with rectangular cross section which originally before rolling contained a ring of twisted filaments. 6 pieces of this wire were soldered together at the smaller side of the rectangle, see fig.2. Current leads were attached, which were powered with the help of a transformer. In this way transverse currents up to $3 \mathrm{kA}$ in the frequency range $0.1-20 \mathrm{~Hz}$ could be produced. The such obtained test array had a length of about $100 \mathrm{~mm}$. It was in subsequent experiments divided Into $4,8,16,32$ and 64 pieces of $25,12.5,6.25 \mathrm{~mm}$ etc, with a laser beam, in order to eliminate the short circuiting of the transverse resistance due to the twisting of filaments. The test configuration did not allow for an external $B$ field so all measurements were taken under self-field conditions only.

The value of the transverse current was monitored with a Hall probe and a Rogowski coil simultaneously. A pick up coil wound around the test sample perpendicular to the current was used to detect the expected $\mathrm{B}$-component averaged over the width and the thickness of the wire, see fig. 2.

Two types of experiments were performed at each subdivision of the sample

a) a measurement of the decay time of a current starting at about $5 \mathrm{kA}$, using the signal of the Hall probe or the Rogowski coil.

b) measurement of the amplitude ratio and phase shift of the induced magnetic field with respect to the applied sinusoidal current. 


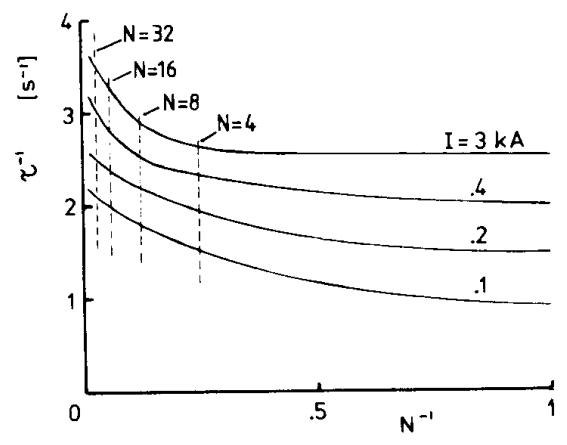

Fig. 3 Measured $\tau$-values as a function of the reciprocal number of subdivisions.

These experiments have been performed at multiples of $0.5 \mathrm{kA}$ amplitude up to $3 \mathrm{kA}$ at frequencies between 0.1 and $20 \mathrm{~Hz}$ in a 1-2-5 sequence. All measurements were taken with a data acquisition system averaging at least 50 periods.

\section{Experimental results}

The results of the decay time experiments are shown in fig. 3. Here $\tau^{-1}$ as a function of the number of subdivisions is plotted at various values of the current. $\tau^{-1}$ is proportional to the total resistance in the secundary loop of the transformer circuit, which consists of the resistances of the current leads and the test sample in series. The change in $\tau^{-1}$ therefore is only due to the change of the resistance in the sample. $\tau^{-1}$ is independent of the current above $1 \mathrm{kA}$. At lower levels $\tau^{-1}$ increases by a factor of 2 , probably due to the fact that now only the outer filaments are involved and the inner filaments, which have a larger series resistance, are not longer taking part in the current conduction. At any current level $\tau^{-1}$ is independent of the number of subdivisions $N$, when $N<16$ in accordance which the fact that the half twistlength, $7.5 \mathrm{~mm}$, is shorter than the length of the wire pieces.

The results for the magnetic measurements are shown in fig. 4 presenting the real part of the averaged $\mathrm{B}_{\mathrm{x}}$ component $\widetilde{\mathrm{B}}_{\mathrm{x}}$ (in phase with the current) and the (out of phase) imaginary part as function of each other. The parameter along each line is the frequency. Different lines are for different current amplitudes. In fig. 5 the influence of the number of subdivisions is shown at constant transverse current $(1 \mathrm{kA})$. As expected at larger $\mathrm{N}$ where the short circuiting of transverse resistance due to the twisting of the filaments is eliminated, the magnetic effect disappears. The change in shape of the curves is due to the three dimensional character of the induced current patterns, which also changes if the cuts come closer to each other. In the mathematical considerations we will pay no attention to this, since it is of no further interest for our conclusions.

\section{Mathematical considerations}

The mathematical modelling of the rectangular strand is relatively simple if we omit the $z$ dependence of the problem, i.e. the solution is valid for an undivided sample much longer than the twistlength $l_{p}$. From symmetry it follows that only one quarter of the cross section has to be considered, see fig. 6. The wire has a width $2 a$ in the $x$ direction, in which also the transverse current flows. The thickness is 2b. Without loss of generality we can take $a=b=1$. The filament region is confined to an infinitisimal thin layer, positioned at $y=\beta b$ and $x=\alpha a$. The filaments are supposed to make an angle $\varphi$ with the $z$-direction and $\pi / 2-\varphi$ with the $x$-direction at $y=\beta b$, see fig. 2 . For the twistlength $l_{p}$ we now find: $l_{p}=4(\alpha a+\beta b) / t a n$ $\varphi$. Note that the twist is taken counterclockwise, The time variation of the variables will be discribed by the factor $e^{i w t}$. The solution now obeys the Helmholtz equations $\Delta \bar{B}=i \omega \mu_{0} \tau \bar{B}$ with a set of boundary and symmetry relations. These are sometimes more easily written in components of $\bar{E}$ which of course are related to $\bar{B}$ by $\nabla \times \bar{B}=\mu_{0} \sigma \bar{E}$

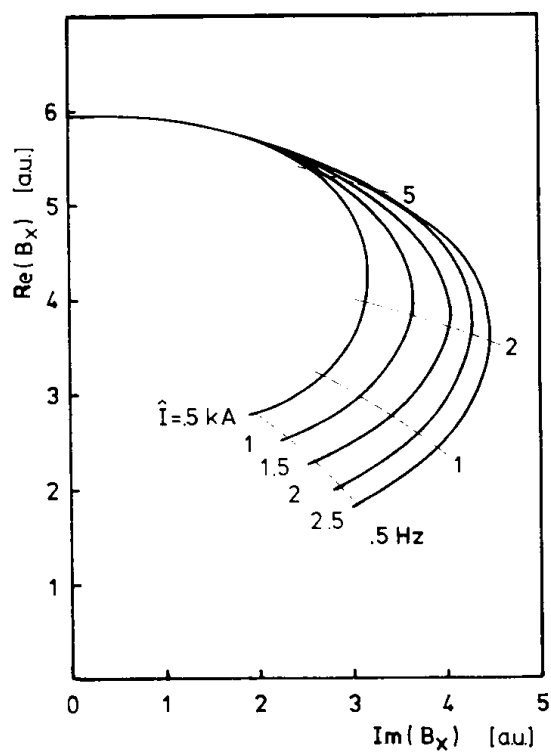

Fig. 4 Measured real and imaginary parts of $\tilde{B}_{x}$ at different amplitudes for the undivided sample. The frequencies are indicated.

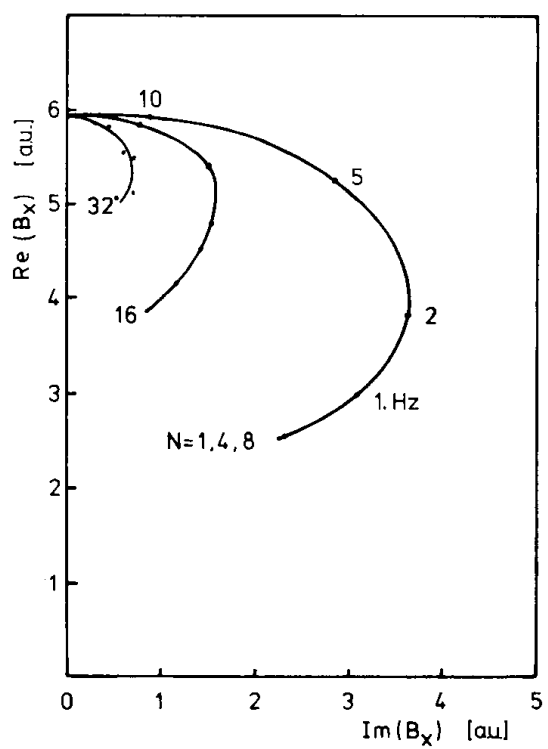

Fig. 5 Measured real and imaginary parts of $\tilde{\mathrm{B}}_{x}$ as function of each other at $1 \mathrm{kA}$ amplitude and different number of subdivisions $N$. The parameter along each curve is the frequency. 


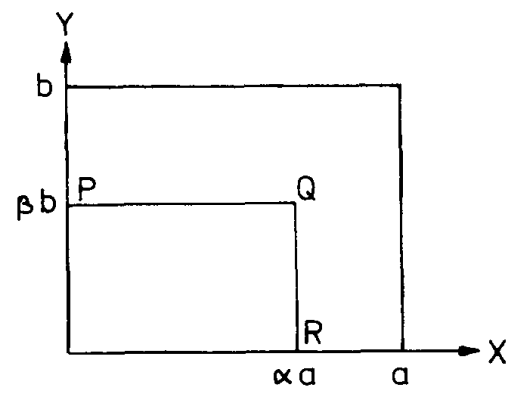

Fig. 6 Geometry of a quarter of the cross section of the wire used in the calculations.

The relations are:

$$
\begin{aligned}
& \partial_{x} B_{x}=0 \text { for } x=0, a \\
& \partial_{y} B_{x}=0 \text { for } y=0 \\
& B_{y}(0, y)=B_{y}(0, x)=B y(a, y)=0 \\
& B_{z}(x, b)=\mu_{0} I_{x} / 2 \\
& B_{z}(x, 0)=0 \\
& \partial_{x} B_{z}(0, y)=\partial_{x} B_{z}(a, y)=0
\end{aligned}
$$

At $y=b \quad B_{x}$ and $B_{y}$ are cont inuous and can be put equal to the periodic solution in vacuum:

$$
\begin{aligned}
& B_{x}(x, y)=\sum_{n=1} C_{n} \cos (n \pi x / a) e^{-n \pi y / a} \\
& B_{y}(x, y)=-\sum_{n=1} C_{n} \sin (n \pi x / a) e^{-n \pi y / a}
\end{aligned}
$$

The coefficients $C_{n}$ can be eliminated by requiring:

$$
\int_{0}^{a} B_{x} \cos (n \pi x / a) d x+\int_{0}^{a} B_{y} \sin (n \pi x / a) d x=0,
$$

which provides now sufficient integral (summation) equations for $B_{x}$ and $B_{y}$ at $y=b$. For more details see P. C. Rem [4].

$$
\text { The twist of the filaments can be }
$$
incorporated by the equations, valid for unsaturated filaments only,

$$
\begin{aligned}
& E_{/ /} \sin \varphi+E_{z} \cos \varphi=0, \\
& E_{/ /}=E_{x}(x, \beta b), \quad x \leq \alpha a, \\
& E_{/ /}=-E_{y}(\alpha a, y), y \leq \alpha a,
\end{aligned}
$$

and

$$
\begin{aligned}
& j_{x} \cos \varphi-j_{z} \sin \varphi=0, \text { at } y=\beta b, \quad x \leq \alpha a, \\
& j_{y} \cos \varphi+j_{z} \sin \varphi=0, \text { at } x=\alpha a, y \leq \beta b,
\end{aligned}
$$

$j_{x}, j_{y}, j_{z}$ being the components of the surface current density in the filament layer. Furthermore we note that $E_{z}$ is a continuous function everywhere, $B_{y}$ and $E_{x}$ are continuous at $y=\beta b, B_{x}$ and $E_{y}$ at $x=\alpha a$. For the solution for $\bar{B}$ and $\bar{j}$ of the above set of equations the dissipated power and the average value of $B_{x}$ can be determined. The dissipated power is proportional to:

$$
P=\operatorname{Re} \int_{0}^{a}\left(E_{z} B_{x}^{*}-E_{x} B_{z}^{*}\right) d x,
$$

( $B$ is the complex conjugate) and the effective resistance to:

$$
\mathrm{R}(\alpha, \beta, \varphi, \omega)=2 \mathrm{P} / \mathrm{I}^{2} .
$$

The average value of $B_{x}$ is defined by:

$$
\tilde{\mathrm{B}}_{\mathrm{x}}(\alpha, \beta, \varphi, \omega)=(1 / \mathrm{ab}) \int_{0}^{\mathrm{a}} \int_{0}^{b} \mathrm{~B}_{\mathrm{x}} \mathrm{dxdy} \text {. }
$$

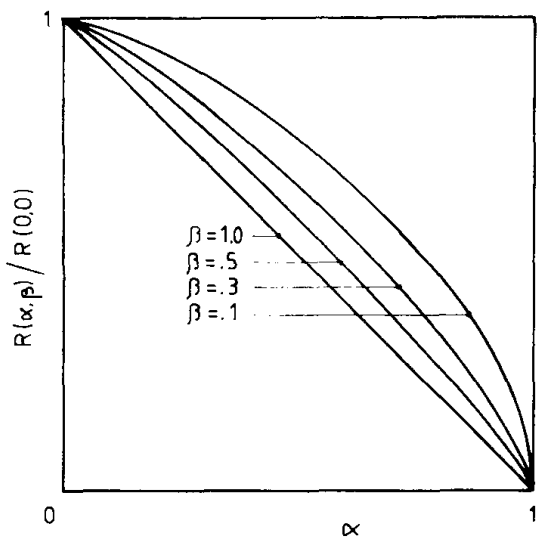

Fig. 7 Calculated DC resistance as a function of $\alpha$ for different $\beta$.

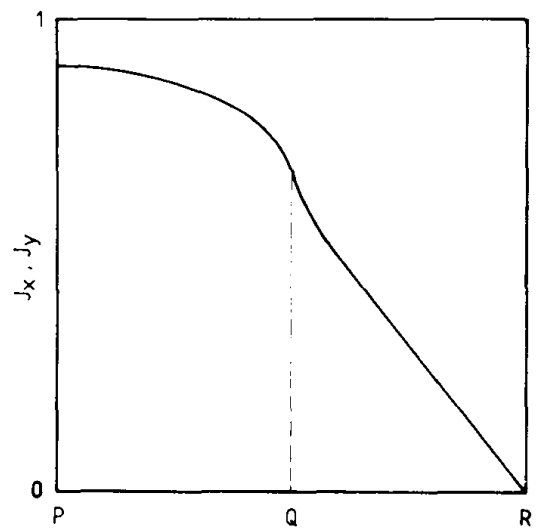

Fig. 8 Calculated transverse components of the the superconcucting surface current: $j_{x}$ along $P Q$ and $-j_{y}$ along $Q R$ (see fig. 6).

\section{Numerical results}

For $\omega=0, \widetilde{\mathrm{B}}_{\mathrm{x}}$ is a real constant and no voltage, proportional to $\omega \widetilde{B}_{x}$, is detected by the pick up coll. The, now DC, resistance $R$ depends only on $\alpha$ and $\beta, i . e$. the position of the filament layer and not on the twist angle $\varphi>0$. In case of no twist $(\varphi=0)$ however, nor a decrease in resistance, neither a $\widetilde{B}_{x}$ value is expected. The relative decrease of $R(\alpha, \beta, \varphi, 0)$ is shown in fig. 7. Fig. 8 shows the surface current density in the layer at some twist angle. Although the resistance $R$ is not effected by $\varphi$ the current density in the direction of the filaments increase with $(\sin \varphi)^{-1}$ and can take very large values.

For $\omega>0$ our main interest goes to the values of $\tilde{B}_{x}$. In fig. 9, the values of $\omega\left|\tilde{B}_{x}\right|$ are plotted as a function of $\omega^{1 / 2}$. (At high frequencies the skineffect will dominate the contribution of the filament layer). Characteristic peaks in $\omega\left|\widetilde{B}_{x}\right|$ occur depending on the twistangle (or the twist length). In fig. 10 the decrease of $R(\alpha, \beta, \varphi, \omega)$ is shown $(\alpha=\beta=1 / 2)$. At small twist angles this decrease is only present at small values of $\omega$. Finally in fig. 11 the real and imaginary part of $\tilde{B}_{x}$ are plotted against each other for different $\varphi$ values. The calculated curves agree satisfactory with the observed ones of $\mathrm{fig} .4$. 


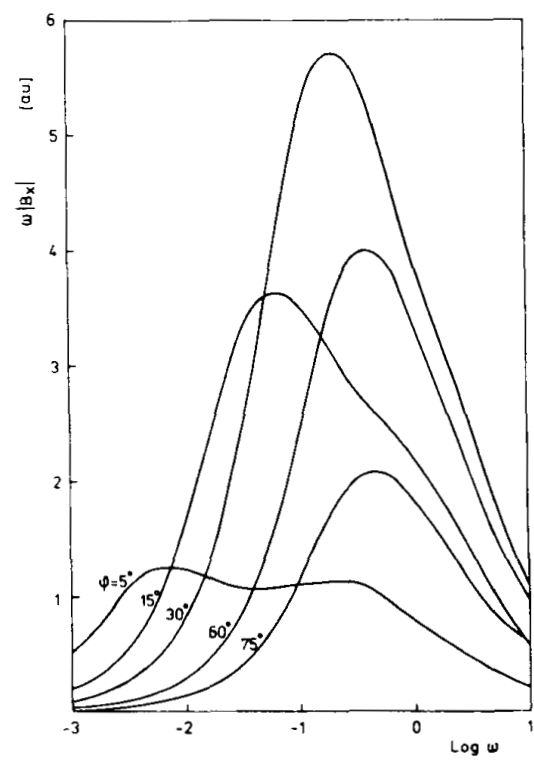

Fig. 9 Calculated amplitude of the induced voltage as a function of $\log \omega$ for different values of $\varphi ; \alpha=\beta=1 / 2$.

\section{Conclusions}

The theory regarding the effective transverse resistivity of soldered strands has been extended to the whole $\mathrm{AC}$ domain. An accompanying magnetic effect due to the twist of the filaments has been measured and qualitatively explained by a simple model of the filament layer in the strands. From the results the suggestion arises that the coupling current experiences maximum resistivity if the strands are almost untwisted. More detalled analysis is necessary, since aiso the other component of the constitutive equations and the saturation effects, which certainly occur in varying fields, have to be taken into account.

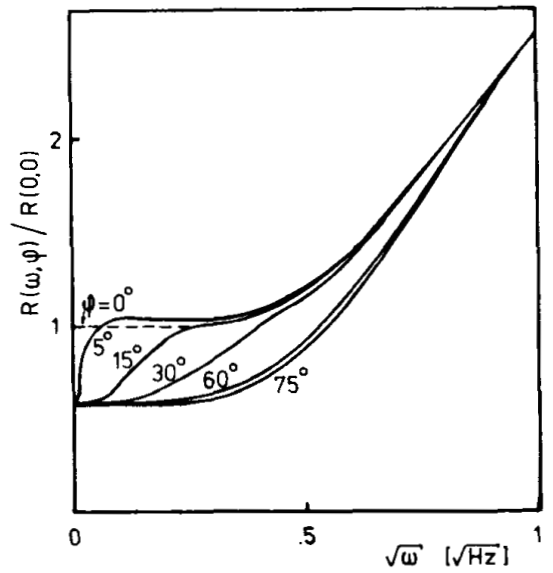

Fig. 10 Calculated relative AC transverse resistance for different twist angles $\varphi$ as a function of

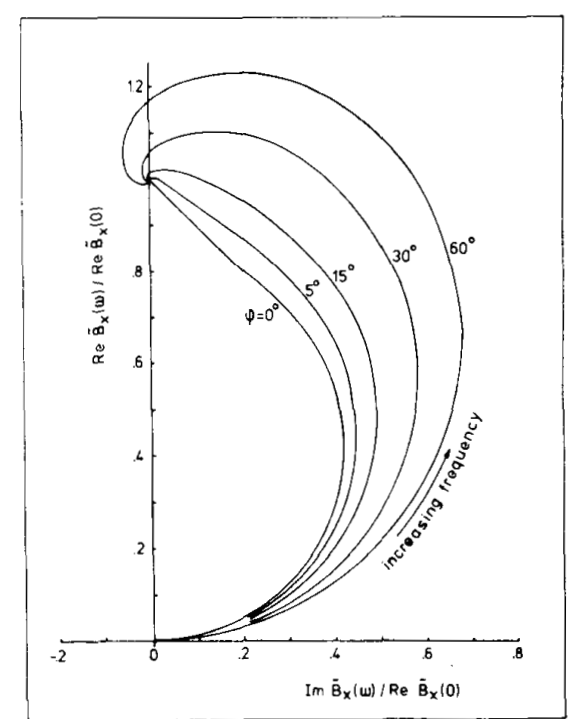

Fig. 11 Calculated real and imaginary parts of $\tilde{B}_{x}$ at different twist angle $\varphi ; \alpha=\beta=1 / 2$. The parameter along each curve is $\omega$.

\section{References}

[1] V M. Dobrin, et al. "Direct measurement of the transverse resistivity of mult ifilament superconducting composites." Cryogenics, vol 22, pp. 115-119, March 1982

[2] K. Kwasnitza, et al. " Large AC losses in superconducting multistage $\mathrm{Nb}_{3} \mathrm{Sn}$ cables due to low transverse resistance," in Proceedings of ICEC 11. 1986, pp. 741-745.

[3] J. A. Eikelboom and J. A. Roeterdink, "AC-losses in the ECN concept of the NEI IF conductor", paper CR-10, this conference.

[4] P. C. Rem, Numerical models for $A C$ superconductors. Thesis, University of Twente, 1986, pp. 112-114. 